\title{
Actuators Placement for Active Dam- ping of Vibration on Two Dimensional Clamped Plate
}

\author{
Peter Šolek (SK) peter.solek@stuba.sk \\ Martin Horínek(SK) martin.horinek@stuba.sk
}

\section{BIOGRAPHICAL NOTES}

Peter Šolek, prof. Ing. PhD. he graduated from Slovak Technical University in Bratislava, Instrumentation field of study, Regulation and automation technology. After graduation he became an internal aspirants at Department of Technical Mechanics Faculty of Mechanical Engineering STU and defended his dissertation in 1978. In 1992 he defended his habilitation work and became an Associate Professor in Applied Mechanics. Since 2007, acts as head of the Institute of Applied Mechanics and Mechatronics Engineering at University of Technology in Bratislava. Is a member of trade union committees in the fields of study Mechatronics and Manufacturing machinery and equipment. He is a member of several professional organizations at home and is Chairman of the National Committee for the IFTOMM. He is the author of one monographs and co-author of nine CC publications. As a responsible person and deal with addresses APVT and VEGA projects and working in an international project CEEPUS between EU and U.S. universities. In 2010 he became Professor of Mechatronics.

Martin Horínek, Ing. graduated at Slovak Technical University in Bratislava, Faculty of mechanical engineering. Bachelor degree in mechatronics, master degree in applied mechanics. Currently PhD student in field mechatronics.

\section{KEY WORDS}

Actuator Placement, Controllability, Norms, Two-Dimensional Flexible System

\section{ABSTRACT}

This article deals with the investigation of optimal actuator placement for two dimensional systems. In this article the two dimensional system is represented by a clamped plate. The intention of optimal actuator placement is to place actuators in minimal number of actuators to ensure controllability of the system. In this article is compared placement of force actuators and piezoelectric actuators. The approach proposed in this article is based on the evaluation of the $\mathrm{H}_{2}$ and $\mathrm{H}_{\infty}$ norms. The optimal actuator placement satisfies the condition of controllability.

\section{INTRODUCTION}

For the target of improving the performance control of flexible structures, it is useful to investigate various actuators and sensors location. The first purpose of the investigation is to determine the minimal number of actuators and sensors and to meet requirements of controllability, observability and spillover prevention. Second purpose is that the minimal subset of actuators and sensors has the same controllability and observability properties as the original set. The importance of actuator and sensor placement 
is supported in many investigations and contributions. The articles $[1,2]$ used the norms $\mathrm{H}_{2}, \mathrm{H}_{\infty}$ and Hankel singular values for the actuator and sensor placement. The contributions [3, 4] use observability and controllability grammians for the actuator and sensor placement. Next big group of articles use the various formulations of optimization problems $[5,6]$ for the solution of the actuator and sensor placement the flexible structures.

\section{Conrollability and observability}

A structure is controllable if the applied actuators excite all its structural modes. A structure is observable if the realized sensors detect the motion of all the modes. This information is limited, and answers of mode excitation or detection are in terms yes or no. The alternative approach uses grammians to determine the system properties. Grammians express the controllability and observability properties qualitatively and they are nonnegative matrices. The definitions of the controllability and observability grammians are in the form:

Where $(\mathrm{A}, \mathrm{B}, \mathrm{C})$ is the state-space representation

$$
\begin{aligned}
& W c(t)=\int_{0}^{t} \exp (A \tau) B B^{T} \exp \left(A^{T} \tau\right) d \tau \\
& W o(t)=\int_{0}^{t} \exp \left(A^{T} \tau\right) C^{T} C \exp (A \tau) d \tau
\end{aligned}
$$

of a time continuous system. We can express the controllability and observability grammians conveniently from the next differential equations (3) and (4)

$$
\begin{aligned}
& \dot{W}_{c}=A W c+W c A^{T}+B B^{T} \\
& \dot{W}_{O}=A^{T} W o+W o A+C^{T} C
\end{aligned}
$$

The solutions $W c(t)$ and $W o(t)$ are time-varying matrices. For a stable system, we obtain the stationary solutions of the above equations by assuming

$$
\dot{W}_{c}=\dot{W}_{O}=0
$$

Now the differential equations (6) and (7) are replaced with the algebraic equations, called Lyapunov equations

$$
A W c+W c A^{T}+B B^{T}=0
$$

$$
A^{T} W o+W o A+C^{T} C=0
$$

\section{Norms}

System norms serve as a measure of intensity of its response to standard excitations, such as unit impulse or white noise of unit standard deviations. The standardized response allows comparing different systems. For flexible structures the $\mathrm{H}_{2}$ norm has an additive property - this property is a root-meansquare sum of the norms of individual modes. All norms of a mode with multiple inputs (outputs) can be decomposed into the rms sum of norms of a mode with a single input (output). When (A, B, $\mathrm{C})$ is a system state-space representation of a linear system and let $G(\omega)=C(j \omega I-A)^{-1}$ is the transfer function. The $\mathrm{H}_{2}$ norm is formulated in form (8)

$\|G\|_{2}^{2}=\frac{1}{2 \pi} \int_{-\infty}^{\infty} \operatorname{tr}\left(G(\omega)^{*} G(\omega)\right) d \omega$

A suitable way to determine the $\mathrm{H}_{2}$ norm is using the formula (9)

$\|G\|_{2}=\sqrt{\operatorname{tr}\left(C^{T} C W c\right)}=\sqrt{\operatorname{tr}\left(B B^{T} W o\right)}$

where $W c$ a $W o$ are the controllability and observability grammians.

The $\mathrm{H}_{\infty}$ norm is formulated as (10)

$\|G\|_{\infty}=\sup \frac{\|y(t)\|_{2}}{\|u(t)\|_{2}}$

where $y(t)$ is the system output and $u(t)$ is the system input.

\section{Actuator placement}

Actuators and sensors placement are solved independently and both procedures are similar. Indicate by $G$ the transfer function with all $S$ candidate actuators. The index of placement $\sigma_{2 \mathrm{ki}}$ that evaluates the $k$-th actuator at the $i$-th mode in terms of the $\mathrm{H}_{2}$ norm is defined with respect to all the modes and all admissible actuators (11).

$\sigma_{2 k i}=w_{k i} \frac{\left\|G_{k i}\right\|_{2}}{\|G\|_{2}}$

where $\mathrm{w}_{k i} \geq 0$ is the weight assigned to the $k$-th actuator and the $i$-th mode, $\mathrm{n}$ is the number of modes, $G$ ki is the transfer function of the $i$-th mode and $k$-th 
actuator.

The index of placement $\sigma_{\text {»ki }}$ evaluates the $k$-th actuator at the $i$-th mode in terms of the $\mathrm{H}_{\infty}$ norm. This index is defined for all modes and all admissible actuators (12).

$\sigma_{\infty k i}=w_{k i} \frac{\left\|G_{k i}\right\|_{\infty}}{\|G\|_{\infty}}$

The matrix of placement gives an insight into the placement properties of each actuator because the index of placement of the $k$-th actuator is defined as the rms sum of the $k$-th column.

In case of the $\mathrm{H}_{2}$ norm, it is the rms sum of the k-th sensor indices over all modes (13).

$$
\sigma_{s k}=\sqrt{\sum_{i=1}^{n} \sigma_{i k}^{2}}
$$

And in the case of $\mathrm{H}_{\infty}$ norm it is (14).

$$
\sigma_{s k}=\max _{i}\left(\sigma_{i k}\right)
$$

\section{Example}

Using $\mathrm{H}_{\infty}$ and $\mathrm{H}_{2}$ norms for determining optimal actuator placement is presented in the following example dealing with a clamped plane plate which is shown in Fig. 1. Calculation of natural frequencies and modes of the plate was done using finite element methods in program Ansys. Analyzed model of plate has six degrees of freedom in each node: displacements in directions $\mathrm{x}, \mathrm{y}, \mathrm{z}$ and rotations around these directions. The length of the plate is $50 \mathrm{~cm}$, width is $40 \mathrm{~cm}$ and its thickness is $2,5 \mathrm{~mm}$.

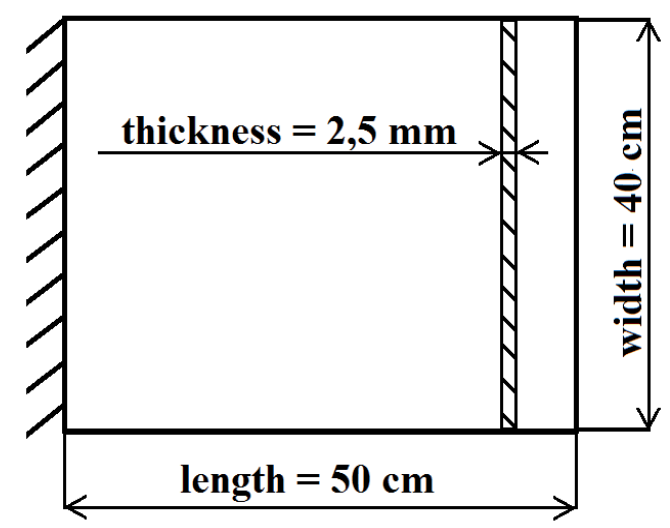

Fig. 1 Scheme of the clamped plate
When a force actuator for suppressing an amplitude of vibration is used shown in Fig. 3 , then it is needed to be placed perpendicularly to the surface of the plate for damping the motion. Using the above presented $\mathrm{H}_{\infty}$ norm placement technique finds the best place for actuators functioning in direction perpendicular to the surface of the plate to control the first, second, third, and fourth own mode and to control simultaneously first four modes.
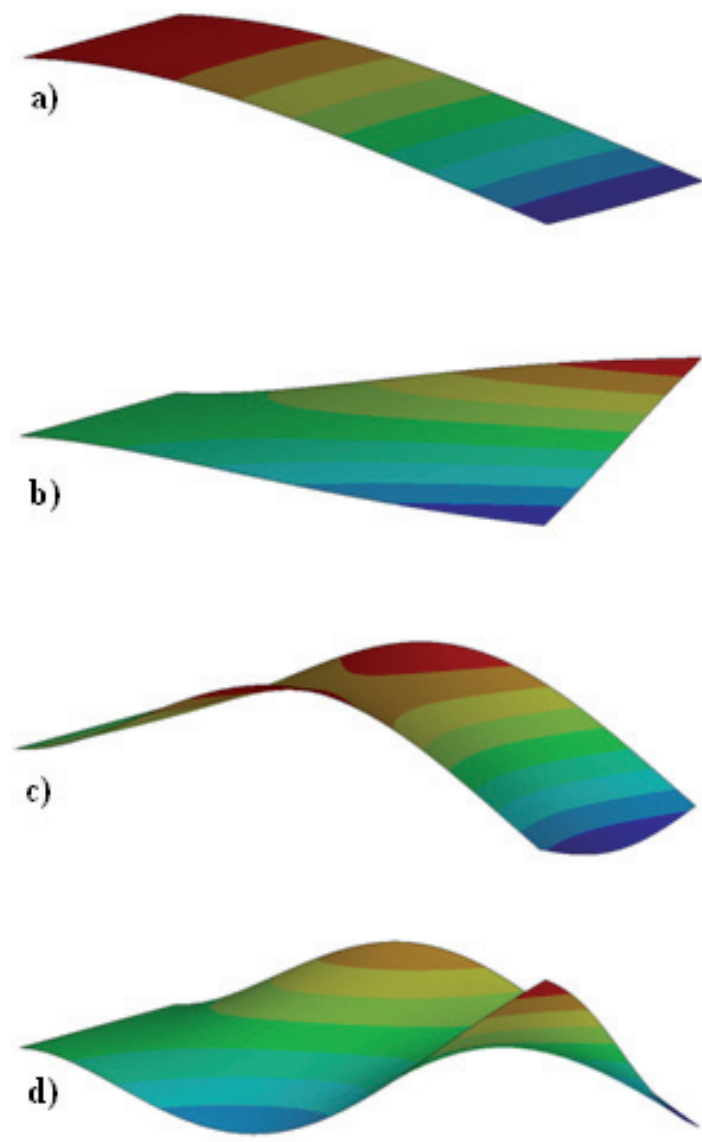

Fig. 2 Eigenmodes of the clamped plate presentation. a) first mode, b) second mode, c) third mode, d) fourth mode

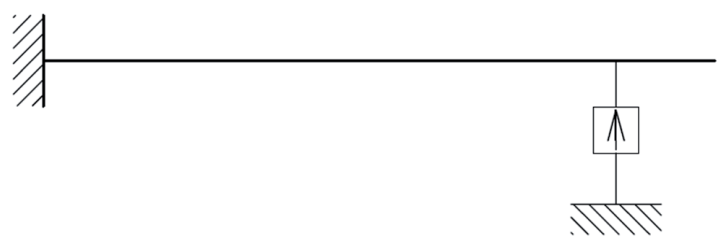

Fig. 3 Applying force on the structure 


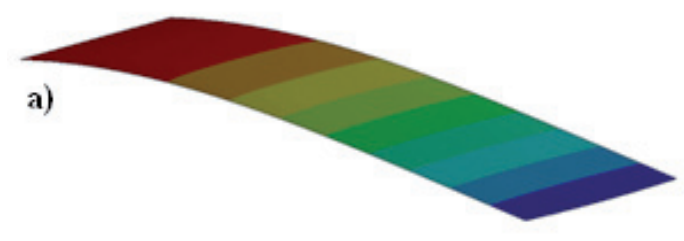

b)
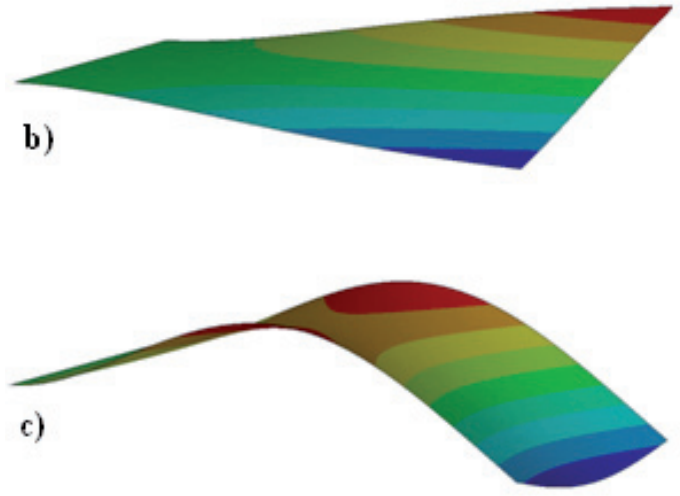

Fig. 4 Actuator placement indices as a function of actuator locations: a) for the first mode, b) for the second mode, c) for the third mode, d) for the fourth mode

We obtain $\mathrm{H}_{\infty}$ norm Gki $\omega_{\infty}$ for the kth mode $(\mathrm{k}=$ $1,2,3,4)$ and ith actuator location. From these norms we obtain the actuator placement indices for each mode from (12), using weight such that maxi $\left(\sigma_{\text {ski }}\right)=1$. The plots of $\sigma_{\text {×ki }}$ are shown in Fig. 4. The plot of the actuator placement indices for the first mode in Fig. 4a) shows the maximum in the middle of the shorter free edge, and that indicates that an actuator shall be placed at that place. The same situation appears in the third mode, although the first and third modes are different which is shown in Fig. 4c). In Fig. 4b) and 4d) indices for second mode and fourth mode reach their maximal values on two free corners of the plate.

Next, we determine the indices for actuator placing for controlling first four modes according to (14) $\sigma_{\infty 1234 \mathrm{i}}=\max \left(\sigma_{\infty 1 \mathrm{i}}, \sigma_{\infty 2 \mathrm{i}}, \sigma_{\infty 3 \mathrm{i}}, \sigma_{\infty 4 \mathrm{i}}\right)$, we obtain three maxima on free edge of the plate, which is shown in Fig. 5 a).

For determining the best actuator placement, the $\mathrm{H}_{2}$ norm $\sigma_{\text {ki2 }}$ for the kth mode $(\mathrm{k}=1,2,3,4)$ and ith actuator location was used for solving this example as well. From these norms and using (11) we obtain indices for each individual mode and from the equation (13) we determine the indices for the first four modes. Using $\sigma_{2,1234 i}$ we obtain indices with two maxima on shorter free edge, and these places are the most suitable for the actuator placement, shown in Fig. 5b).

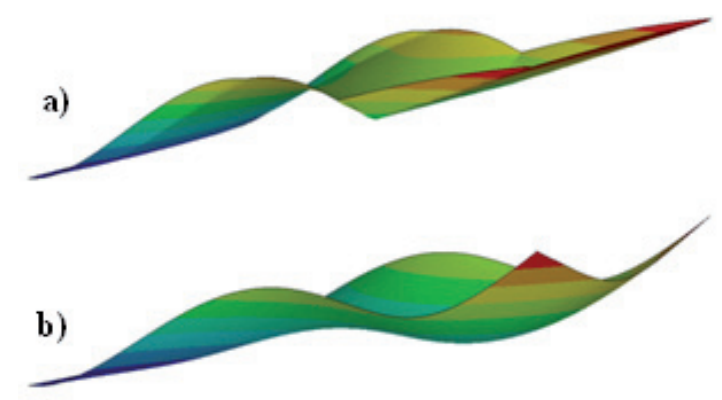

Fig. 4 Actuator placement $\mathrm{H} \infty / \mathrm{H} 2$ indices as a function of actuator location. a) for the first four modes of norm $\mathrm{H}_{\infty}$, b) for the first four modes of norm $\mathrm{H} 2$

Also piezoelectric actuators are suitable for using in active vibration suppression. This kind of actuator is shown in Fig. 6 . Such a piezoelectric actuator acts against deformation on an outer layer of the structure during vibration.

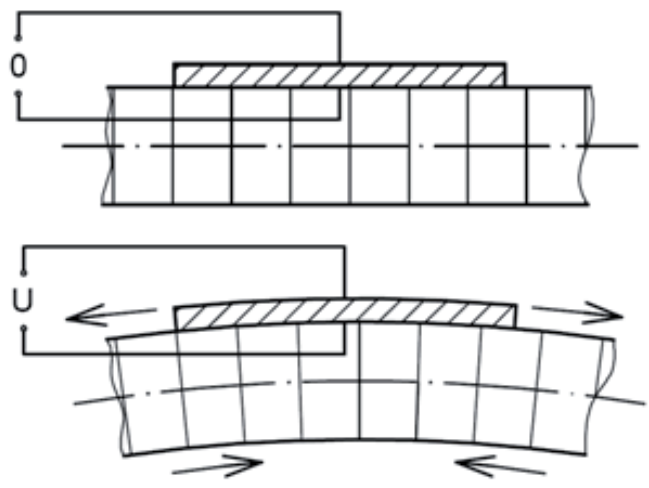

Fig. 6 Applying piezoelectric actuator on the structure

In Fig. 7 there are shown maximal strain during first four single own modes of the plate clamped on one edge. These maximal strains are indices for placing actuators for decreasing a strain during vibration. As we can see actuator placement indices are maximal in the clamped edge but for every mode in a different place. For the first mode which is shown in Fig. 7 a) the maximal index for actuator placement is in the middle of a clamped edge. In Fig. 7 b), c), d) is shown that indices for actuator placement for second, third, and fourth mode are approximately on the same places on the clamped edge. 

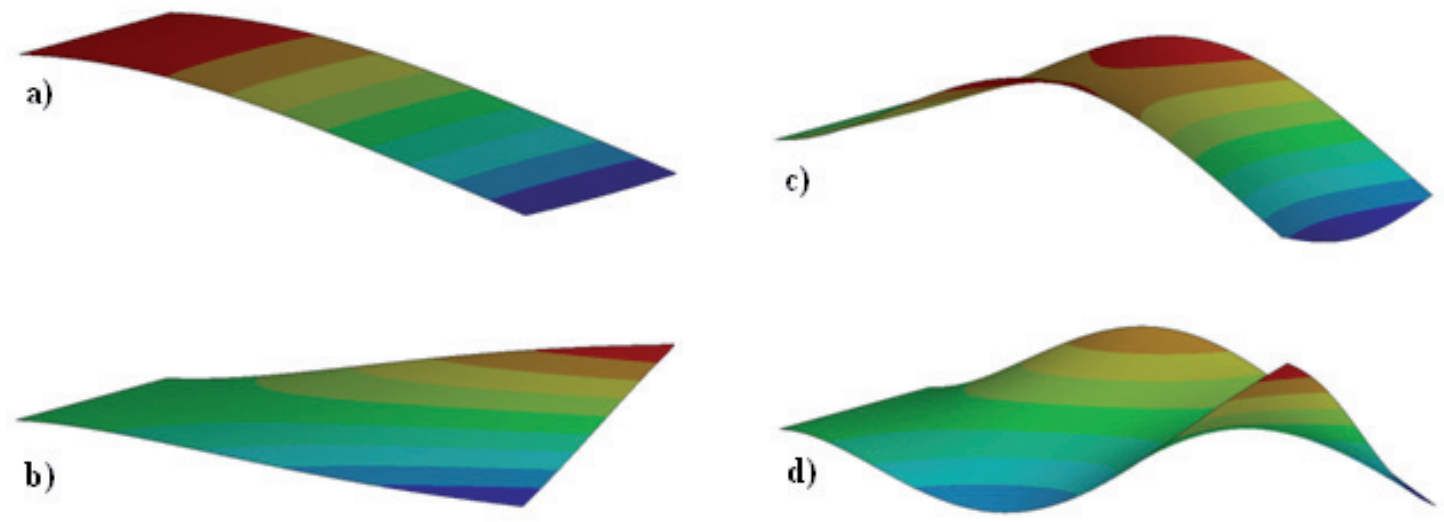

Fig. 7 Actuator placement indices as a function of actuator locations: a) for the first mode, b) for the second mode, c) for the third mode, d) for the fourth mode

$\mathrm{H}_{\infty}$ norm for first four modes according to $\sigma_{\infty 1234 i}$ $=\max \left(\sigma_{\infty 1 \mathrm{i}}, \sigma_{\infty 2 \mathrm{i}}, \sigma_{\infty 3 \mathrm{i}}, \sigma_{\infty 4 \mathrm{i}}\right)$ shows in Fig. 8a) three places are on clamped edge for piezoelectric actuator placing. For the $\mathrm{H}_{2}$ norm using $\sigma_{2,1234 i}$ there are indices with two maxima on a clamped edge, so these places are the most suitable for the actuator placement which is shown in Fig. 8b).

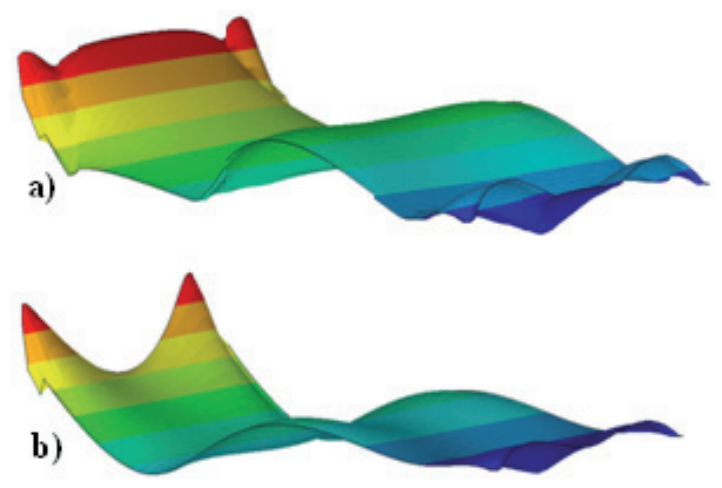

Fig. 8 Actuator placement $\mathrm{H}_{\infty} / \mathrm{H} 2$ indices as a function of actuator location. a) for the first four modes of norm $\mathrm{H}_{\infty}$, b) for the first four modes of norm $\mathrm{H} 2$

\section{Conclusion}

When force actuators for decreasing amplitude of vibration are placed according to indices of the norm $\mathrm{H}_{\infty}$ for the first four modes, then there are three actuators on the plate on the free edge. In case of actuator placement according to indices of the norm $\mathrm{H}_{2}$ for the first four modes then we have only two actuators on the free corner. For piezoelec- tric actuators there are different location for optimal actuators placement in this boundary condition. For clamped plate, actuators shall be placed on the clamped edge because clamped boundary condition caused maximal strain of outer layer of the plate just on that edge. Evaluated actuator placement according to norm $\mathrm{H}_{\infty}$ when piezoelectric actuators are used there are three places for actuating and for using norm $\mathrm{H}_{2}$ there are two places.

\section{Acknowledgement}

Authors acknowledge the support by the Slovak Grant Agency VEGA -1/4128/07.

\section{References}

[1] Gawronski W. K.: Advanced Structural Dynamics and Active Control of structures. Springer Verlag, New York, 2004

[2] Liu, Wei, Hou, Zhokun, Demetrion, Michael A.: A computationa scheme for the optimal sensor/actuator placement of flexible structures using spatial H2 measures. Mechanical Systems and Signal processing. Volume 20,4, 2006, pp. 881-895

[3] Hiramot K., Doki H., Obinata G: Optimal sensor/actuator placement for active vibration control. Journal of Sound and Vibrations. Volume 229,5,2000, pp. 1057-1075

[4] DeLorenzo M.L.: Sensor and actuator selsction for large space structure control. Journal of Guidance, Control and Dynamics. Vol.13, 1990, pp. 249-257

[5] Kim Y., Junkins J. L.: Measure of control- 


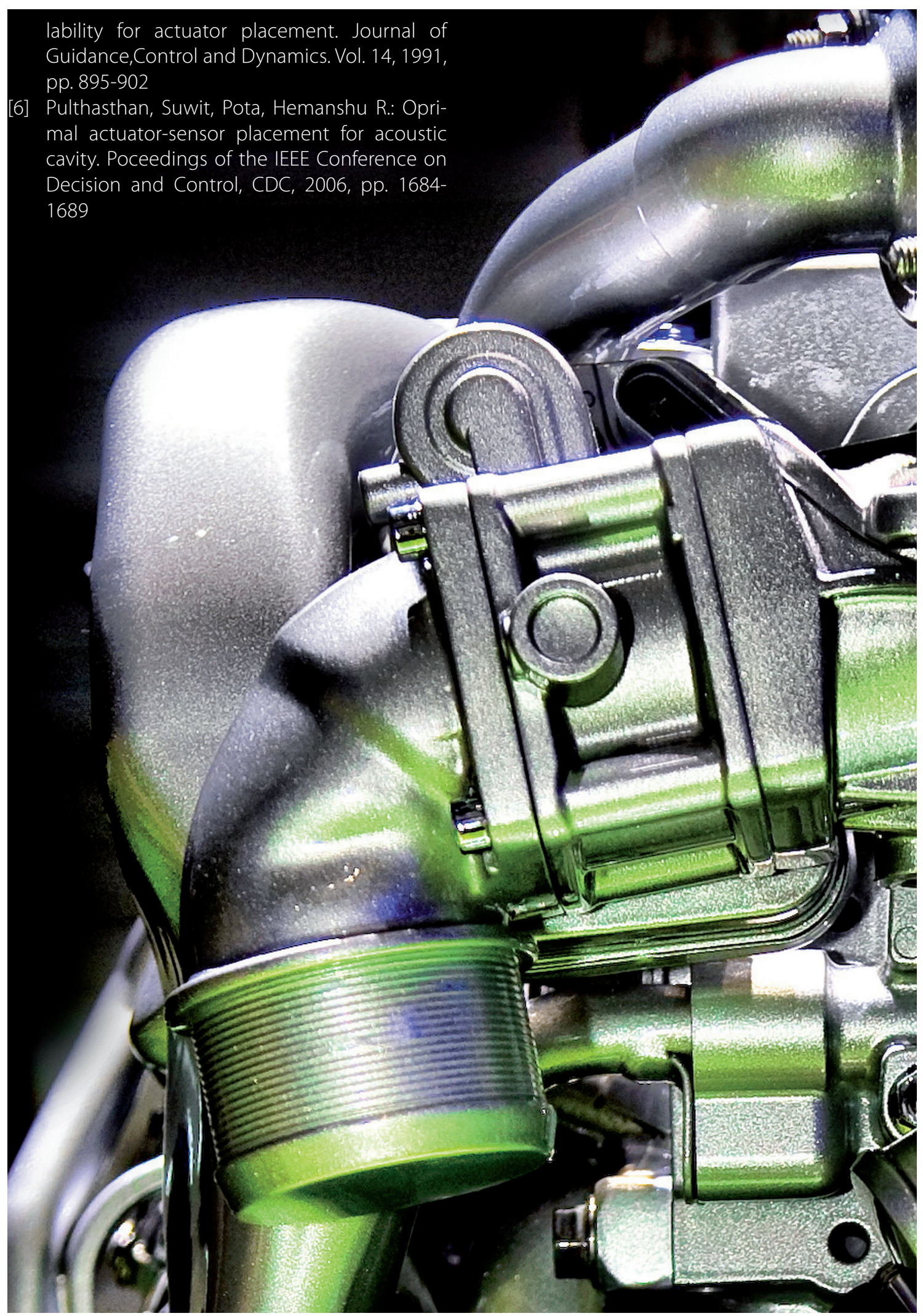

